\title{
Charge Allocation for Hybrid Electrical Energy Storage Systems
}

\author{
Qing Xie ${ }^{1}$, Yanzhi Wang ${ }^{1}$, Younghyun Kim² ${ }^{2}$ Naehyuck Chang ${ }^{\dagger}$, and Massoud Pedram ${ }^{1}$ \\ ${ }^{1}$ University of Southern California, CA, USA, ${ }^{2}$ Seoul National University, Korea \\ 1\{xqing, yanzhiwa, pedram\}@usc.edu, 2\{yhkim, naehyuck\}@elpl.snu.ac.kr
}

\begin{abstract}
Hybrid electrical energy storage (HEES) systems, composed of multiple banks of heterogeneous electrical energy storage (EES) elements with their unique strengths and weaknesses, have been introduced to efficiently store and retrieve electrical energy while attaining performance metrics that are close to their respective best values across their constituent EES elements. This paper is the first paper to formally describe the charge allocation problem and provide a systematic solution method aiming at the maximum charge allocation efficiency, which performing proper distribution of the incoming power to selected destination banks. We introduce a generalized HEES architecture and build the corresponding electrical circuit models of the chargers and banks. We formulate a mixed integer nonlinear optimization problem, where the objective function is the global charge allocation efficiency, and the constraints are energy conservations, with careful consideration of the conversion power loss in the chargers, rate capacity effect and self-discharge of the EES elements, charge transfer losses, and so on. We present a rigorous algorithm to achieve a near-optimal global charge allocation efficiency for long-term charge allocation process (i.e., tens of hours.) Experimental results based on a photovoltaic cell array as the incoming power source and a HEES system comprised on batteries and supercapacitors demonstrate a significant gain in charge allocation efficiency for the proposed algorithm.
\end{abstract}

\section{Categories and Subject Descriptors}

C.3 [Special-purpose and Application-based Systems]: Real-time and embedded systems

\section{General Terms}

Algorithms, Design, Management

\section{Keywords}

Hybrid electrical energy storage system, charge management, charge allocation

\footnotetext{
${ }^{*}$ This work is supported by the Brain Korea 21 Project, IC Design Education Center (IDEC), and Mid-career Researcher Program through NRF grant funded by the MEST (No. 2010-0017680). The ICT at Seoul National University provides research facilities for this study.

${ }^{\dagger}$ Corresponding author
}

Permission to make digital or hard copies of all or part of this work for personal or classroom use is granted without fee provided that copies are not made or distributed for profit or commercial advantage and that copies bear this notice and the full citation on the first page. To copy otherwise, to republish, to post on servers or to redistribute to lists, requires prior specific permission and/or a fee.

CODES+ISSS'11, October 9-14, 2011, Taipei, Taiwan.

Copyright 2011 ACM 978-1-4503-0715-4/11/10 ...\$10.00.

\section{INTRODUCTION}

Electrical energy is a high quality form of energy [1] in the sense that it can easily and efficiently be converted into other forms of lower quality energy. Electrical energy consumption generally fluctuates over time according to variation of the load demands (e.g., an information processing or computing system that is running different applications at different times.) Conventional fossil fuel and nuclear power plants can generate a steady amount of power but the rate at which the power generation can be ramped up or down is low. At the same time, the output power levels of most renewable power sources are largely dependent on environmental factors (e.g., the irradiance level or climate conditions) and thereby not controllable. Therefore, electricity generation and consumption are typically not matched with each other. Storage of excess energy avoids energy waste and mitigates over-investment in power generation facilities by shaving the peak power demand. Electrical energy storage (EES) systems thus increase availability of the electrical energy, mitigate the supply-demand mismatches, and reduce the generation capacity required to meet the peak-power demand.

Some actual deployment of grid-scale EES systems (including both homogeneous and hybrid) to mitigate the gap between the supply and demand has been described in [2, 3, 4]. However, current EES systems are mainly homogeneous, that is, they consist of a single type of EES element, and therefore, suffer from a fundamental shortcoming that will plague every homogeneous EES: key metrics (normalized with respect to capacity) of any homogeneous EES cannot be better than those of its individual storage elements.

Let's consider the memory organization in a computer system to draw analogies to what we propose. Although intensive research has focused on improving the memory technologies, it is unlikely that a single type of memory will dominate memory systems because no single type of memory can fulfill all the desirable requirements such as low access delay, high density, low cost, non-volatility, and low power consumption. A practical solution to overcome this problem has been the use of memory hierarchy comprising of heterogeneous types of memory devices that can hide drawbacks of each memory type while realizing its benefits. A well designed and controlled memory hierarchy provides the microprocessor with a memory resource that has the capacity of its largest component, a level of performance that is close to that of its fastest component, at a per-bit cost that is close to that of its least expensive component.

In the same way, a homogeneous EES system approach is not desirable because none of the existing types of EES elements can fulfill all the required performance metrics such as power density, energy density, cost per unit capacity, weight per unit capacity, round-trip efficiency, cycle life, and environmental effects. This limitation prevents the adoption of a wide range of socially and economically useful technologies, such as widespread adoption of 
grid-scale EES and electric vehicles (EVs), while causing significant inefficiencies in many technologies. Hence, elimination of this limitation of homogeneous EES systems is the primary motivation for our research.

A hybrid EES (HEES) system is consisting of different types of EES elements[5, 6], where each type has its unique strengths and weaknesses. The HEES system can exploit the strength of each type of EES element and achieve a combination of performance metrics that is superior to that of any of its individual EES components. Based on the properties of the HEES system and characteristics of power sources (or load devices), we also develop corresponding control policies to operate HEES system properly to achieve a near-optimal performance. Inspired by memory allocation policy that are widely used in computing systems, we propose charge allocation policy aiming to maximize the charge allocation efficiency by properly distributing power of the incoming power to selected destination banks. More precisely, we see to answer the following questions: i) what is the optimal voltage level for the charge transfer interconnect (CTI), ii) among all the possible destination EES banks, which one(s) should be selected, and iii) among the selected destination EES banks, how to allocate the charging currents to achieve optimal global charge allocation efficiency for the whole system over a given time period. The charge allocation efficiency is determined by the types of the selected banks and the magnitudes of the charging currents, state of charges (SoCs) of the banks and characteristics of the external power source. Since SoCs of EES banks and source power are time dependent, our solution to charge allocation problem should be online.

In this paper, we introduce a generalized HEES architecture comprised of two EES elements (batteries and supercapacitors) and build the corresponding electrical circuit models for power supplies, chargers, battery banks and supercapacitor banks. We define global charge allocation efficiency as the ratio of energy received by EES banks and the total energy provided by power sources over a given time period. In addition to the energy received by EES banks, we also consider the power dissipations on internal resistances of battery bank and supercapacitor bank, power loss on charger during power conversion and rate capacity effect of battery banks. Therefore, the global charge allocation efficiency is dependent on the source power profile, magnitude of charging current and SoC of each EES bank.

The global charge allocation (GCA) problem is formulated to optimize the global charge allocation efficiency over a given time period. To solve the GCA problem, we start from the instantaneous charge allocation (ICA) problem, which seeks to optimize the charge allocation efficiency at a specific instance of time. Since the ICA problem is essentially a mixed integer non-linear optimization problem (MINLP), which is NP-complete, we propose an effective way of solving the ICA problem and get near-optimal solution in an iterative manner, where in each iteration we solve a convex optimization problem which can be solved in polynomial time. Near-optimal solutions of the original GCA problem can be obtained by incorporating time-dependent constraints for charging currents of different sets of EES banks in the ICA problem, and subsequently, solving the ICA problem with the imposed constraints at every decision epoch throughout the charge allocation process. Such constraints force the charge allocation manager to "consider the future energy production profile", and thereby, avoid greedy decisions that may prove wrong e.g., over charging of certain (high efficiency) EES banks (such a scheme may result in efficiency degradation if some EES banks are already full.) Experimental results show that the percentage improvement from various baseline setups ranges from $8.6 \%$ to $51.4 \%$.

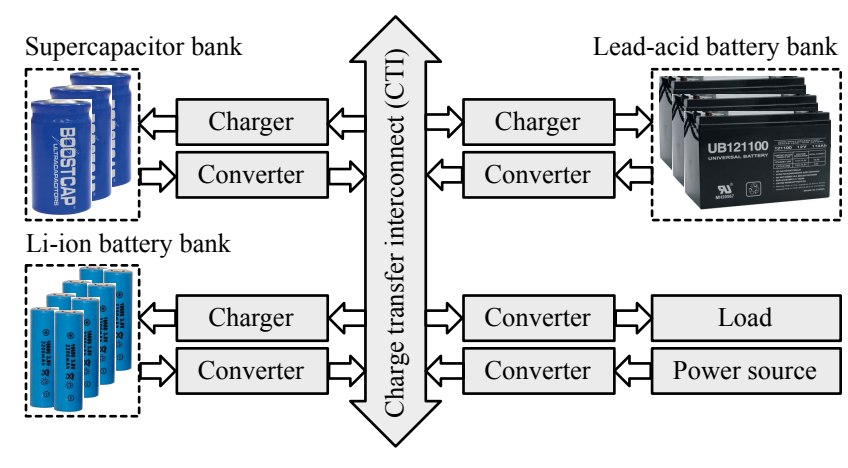

Figure 1: Architecture of the proposed HEES system.

\section{HEES SYSTEMS}

\subsection{HEES System Architecture}

Figure 1 illustrates a conceptual drawing of the proposed HEES system architecture. The system comprises of a number of different EES banks, connected to each other through distributed power converters (i.e., distributed charging and DC/DC conversion circuitry) and CTI. The power converters and CTI are needed because of the different state-of-charge (SoC), terminal voltage, and power rating values for each EES bank, which makes it ill advised to directly connect the EES banks to one another [5, 7]. Since a typical EES element has a low voltage rating and a small energy capacity, storage bank itself is composed of a large number of homogeneous EES elements with same SoCs, organized in an appropriately constructed two-dimensional array using series and/or parallel connections.

As we mentioned in Section 1, no existing EES elements can fulfill all the requirements of an ideal energy storage system such as low capital cost, high cycle efficiency, long cycle life, low selfdischarge rate, and high power and energy densities. For example, a Li-ion battery bank provides high energy capacity, low selfdischarge, stable open terminal voltage, and relative low cost, but suffers from a large rate capacity effect at high input/output power levels. In contrast, a supercapacitor bank has superior cycle efficiency, a long cycle life, and capability of dealing with a high peak power demand (of the load devices), but it has small energy capacity and high self-discharge rate. Therefore, heterogeneous EES banks, such as battery and supercapacitor banks, can be used in a complementary manner in an HEES system to exploit the best characteristics (strengths) of each type of EES element while hiding their shortcomings and weaknesses. According to the properties of HEES system and characteristics of power source and load devices, some charge management policies, including charge migration, charge replacement, charge allocation and bank reconfiguration, are needed to achieve better performance [5]. In this paper, we focus on deriving the near-optimal charge allocation control policy.

\subsection{Charge Allocation}

Charge allocation policy is to determine the best-suited EES banks to store the energy that is coming into the HEES system from a predictable power source. In addition, the charge allocation policy sets the amount of charging current for each selected EES bank. More precisely, the optimal charge allocation policy ideally distributes the incoming charge to all the selected destination banks and achieves the highest possible charge allocation efficiency, which implies that the maximum amount of energy can be stored among the EES banks. Although the optimal charge allocation policy may be also related to load devices, it is out of scope of this paper.

Figure 2 shows the conceptual architecture of the charge allocation subsystem in a HEES system. The HEES system contains $N$ 


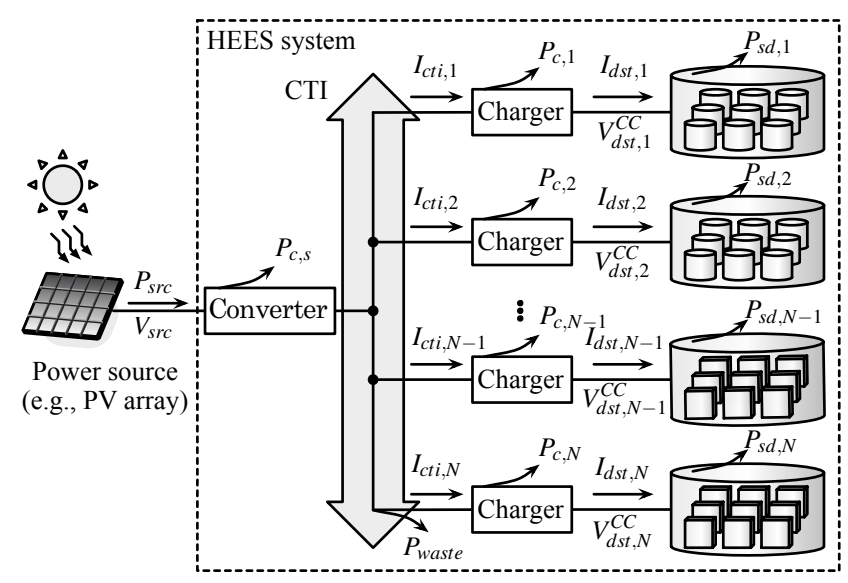

Figure 2: Charge allocation system.

heterogeneous EES banks, denoted by a set $S=\{1,2, \ldots, N\}$, each consisting a number of homogeneous EES elements. Each EES bank is connected to the CTI through a charger (which is a type of power converter.) At any time $t$, a subset $S^{\prime}(t) \subseteq S$ is selected among the $N$ EES banks to receive energy from the power source through the CTI and the intervening chargers.

The proposed charge allocation framework can be applied to any kind of power sources, e.g., photovoltaic array, power grid, windmill, etc. The input power and voltage profile of the source, denoted by $P_{s r c}(t)$ and $V_{s r c}(t)$, respectively, are assumed to be given (or to be predictable.) The power source is connected to the CTI through a source-to-CTI voltage converter with power loss denoted by $P_{c, s}(t)$. This converter supports high voltage and current levels, and can regulate the voltage on the CTI, denoted by $V_{c t i}(t)$, through a feedback loop. The current flows from the source, through the CTI, into (a selected set) of chargers that connect the CTI to destination EES banks. Choosing the optimal $V_{c t i}(t)$ is crucial for the charge allocation problem. For the $k^{t h}$ charger, the input current (known as the charger input current) and output current (known as the charging current) are denoted by $I_{c t i, k}(t)$ and $I_{d s t, k}(t)$, respectively. The charger power loss is denoted by $P_{c, k}(t)$. For the $k^{t h}$ EES bank, the open circuit terminal voltage (OCV) and closed circuit terminal voltage $(\mathrm{CCV})$ are denoted by $V_{d s t, k}^{O C}(t)$ and $V_{d s t, k}^{C C}(t)$, respectively. Generally, $V_{d s t, k}^{O C}(t) \neq V_{d s t, k}^{C C}(t)$ due to the internal resistance and capacitance. The relation between $V_{d s t, k}^{O C}(t)$ and $V_{d s t, k}^{C C}(t)$ is specified in Section 3. $P_{s d, k}(t)$ is the self-discharge power of the $k^{\text {th }}$ destination EES bank, depending on the SoC and bank properties. Notice that if the input power exceeds the maximum receiving capability of the HEES system at time $t$, the excessive input power cannot be stored but dumped to the ground (dissipated as heat.) This amount of power is denoted by $P_{\text {waste }}(t)$, as shown in Figure 2. The precise definition of charge allocation efficiency is presented in Section 3.2.

\section{PROBLEM FORMULATION}

\subsection{System Models}

\subsubsection{Battery bank}

As representative electrical energy storage elements, batteries have advantages of a high energy capacity, a low self-discharge rate, a stable open circuit terminal voltage, and a relatively low cost compared with a supercapacitor. Therefore, battery banks are suitable for long-term and high-energy capacity storage banks. The

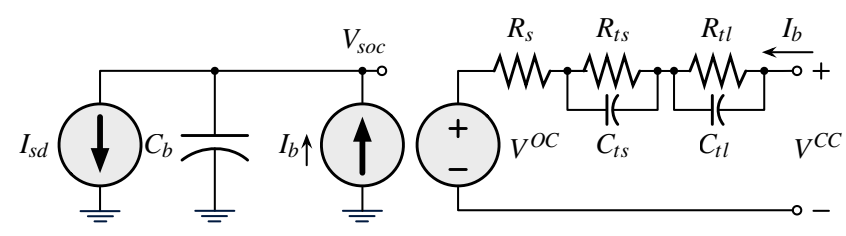

Figure 3: Li-ion battery equivalent circuit model.

proposed charge allocation framework and optimization technique are general in that they can be applied to any types of battery banks, provided that accurate and effective battery models are given. In this paper, without loss of generality, we consider a Li-ion battery to demonstrate the charge allocation problem in this paper.

We adopt the battery model of a Li-ion battery from [8], as shown in Figure 3, which includes a runtime-based model on the left as well as a circuit-based model on the right for accurate capturing of the battery service life and the I-V characteristics. In this model, $C_{b}$ is the remaining charge in the battery, and $V_{S O C}$ is voltaic representation of the SoC of the battery, given by:

$$
V_{S O C}=C_{b} / C_{b, \text { full }} \times 1 \mathrm{~V},
$$

where $C_{b, f u l l}$ is the total charge of battery when it is fully charged. We derive the total charge by converting a nominal battery capacity in Ahr to the amount of charge in Coulomb as follows:

$$
C_{b, f u l l}=3600 \times \text { Capacity } \times \text { Correction_Factor },
$$

where Capacity is the nominal battery capacity in Ahr. Moreover, in Figure $3, I_{b}$ and $I_{s d}$ denote the charging current and the selfdischarging current $\left(I_{s d} \approx 0\right.$ in battery since the self-discharge is negligible), respectively; $V^{O C}$ and $V^{C C}$ are the OCV and CCV of the battery, respectively; $R_{\text {series }}, R_{t s}$ and $R_{t l}$ are internal resistances; and $C_{t s}, C_{t l}$ are internal capacitances. The battery OCV is modeled as a voltage-controlled voltage source of $V_{S O C}$. The other parameters are functions of $V_{S O C}$ as well. The relations are non-linear and given by:

$$
\begin{aligned}
V^{O C} & =b_{11} e^{b_{12} V_{S O C}}+b_{13} V_{S O C}^{3}+b_{14} V_{S O C}^{2}+b_{15} V_{S O C}+b_{16}, \\
R_{\text {series }} & =b_{21} e^{b_{22} V_{S O C}}+b_{23}, R_{t s}=b_{31} e^{b_{32} V_{S O C}}+b_{33}, \\
C_{t s} & =b_{41} e^{b_{42} V_{S O C}}+b_{43}, R_{t l}=b_{51} e^{b_{52} V_{S O C}}+b_{53}, \\
C_{t l} & =b_{61} e^{b_{62} V_{S O C}}+b_{63},
\end{aligned}
$$

where those $b_{i j}$ are empirically parameters [9].

The rate capacity effect of batteries describes that the available charge or discharge capacity decreases with the increase of charge or discharge current. We relate the charging efficiency and the charging current $I_{b}$ using a concave and monotone decreasing function $\eta_{\text {rate }}\left(I_{b}\right)$ [10]. Typically, the rate capacity effect is negligible in supercapacitors, i.e., $\eta_{\text {rate }}=1$.

In reality, the OCV $V^{O C}, \mathrm{CCV} V^{C C}$, charging current $I_{b}$, as well as voltages and currents on internal resistors and capacitors are functions of time $t$. Although the internal resistance and capacitance are also functions of time $t$ (because they are dependent on SoC value), we omit this dependence since they change with time $t$ rather slowly. Therefore, when $V^{O C}(t)$ and $I_{b}(t)$ are given, we derive $V^{C C}(t)$ as follows without loss of generality:

$$
\begin{aligned}
& C_{t l} \cdot d V_{t l}(t) / d t=I_{b}(t)-V_{t l}(t) / R_{t l}, \\
& C_{t s} \cdot d V_{t s}(t) / d t=I_{b}(t)-V_{t s}(t) / R_{t s}, \\
& V^{C C}(t)=V^{O C}(t)+V_{t l}(t)+V_{t s}(t)+I_{b}(t) \cdot R_{S} .
\end{aligned}
$$

Since an EES bank consists of series and parallel connection of a number of homogeneous (and same SoC) EES elements (e.g., bat- 


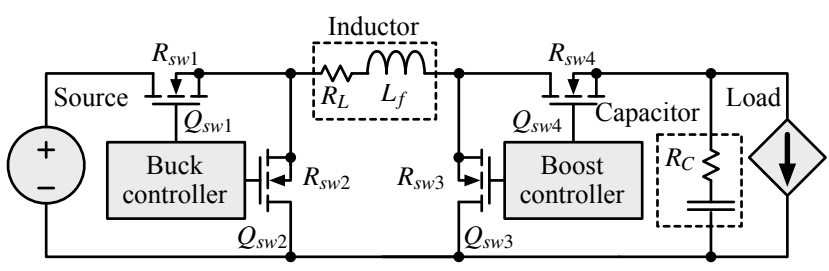

Figure 4: Buck-boost converter circuit model.

teries), the relationship between the bank CCV $V_{d s t, k}^{C C}(t)$ and OCV $V_{d s t, k}^{O C}(t)(1 \leq k \leq N)$ in the charge allocation framework is more involved than the CCV-OCV relation of a single EES element. When the OCV is given (this is typically true since the OCV can be derived from the bank SoC), the $\mathrm{CCV}$ shall be determined separately for each EES element, then combined to obtain the CCV of the bank, considering the topology inside an EES bank. Note that the homogeneity among EES elements will help in deriving the bank CCV. Besides, the self-discharge rate of an EES bank can be calculated in a similar way.

\subsubsection{Supercapacitor bank}

As another representative EES elements, although supercapacitors have lower energy density, it have superior characteristics over batteries or other types of EES elements, in terms of both cycle efficiency and cycle life. Thus, the supercapacitor banks are commonly used to store surplus energy from the battery banks during low load power demand periods, and provide extra energy during peak load power demand periods in HEES systems. The electrical circuit model for the supercapacitor used in this paper contains a low series resistance $(\sim 25 \mathrm{~m} \Omega)$ [9]. Therefore, the following relation between the OCV $V^{O C}(t)$ and the $\mathrm{CCV} V^{C C}(t)$ at time $t$ holds for the supercapacitors such that

$$
V^{C C}(t)=V^{O C}(t)+I_{c}(t) \cdot R_{\text {series }},
$$

where $I_{c}(t)$ is the charging current of the supercapacitor at time $t$.

A primary disadvantage of the supercapacitor is its larger selfdischarge rate compared with other EES elements. A supercapacitor may lose more than $20 \%$ of its stored energy per day even if no load is connected to it [5]. Therefore, for long-term (e.g., tens of hours) charging or discharging operations, the self-discharge effect is not negligible. The voltage decay after $\Delta t$ is given by:

$$
V^{O C}(t+\Delta t)=V^{O C}(t) \cdot e^{-\Delta t / \tau},
$$

where $\tau$ is the self-discharge time constant. For a short time interval $\Delta t$, using Taylor Expansion, the power loss at time $t$ due to selfdischarge can be given by:

$$
P_{s d}(t)=C_{c a p}\left(V_{d s t}^{O C}(t)\right)^{2} / \tau,
$$

where $C_{c a p}$ is the capacitance of the supercapacitor.

\subsubsection{Charger}

A charger is a switching converter that can regulate the output charging current into a desired output charging current value according to our proposed algorithm. In this paper, we use a PWM (pulse width modulation) buck-boost converter model as the charger model, as shown in Figure 4. The input voltage, input current, output voltage and output current of the charger are denoted by $V_{i n}$, $I_{\text {in }}, V_{\text {out }}$ and $I_{\text {out }}$, respectively. Depending on the relation between input voltage $V_{\text {in }}$ and output voltage $V_{\text {out }}$, the charger has two working modes: a buck mode (if $V_{\text {in }}>V_{\text {out }}$ ) and otherwise a boost mode.
When the charger is turned on, the power loss $P_{c}$ of the charger consists of three components: conduction loss $P_{c d c t}$, switching loss $P_{s w}$ and controller loss $P_{c t r l}$ [11], among which the non-zero switching loss and controller loss are independent to the output current $I_{\text {out }}$. When the charger is turned off, the power loss $P_{c}$ is zero because the controller of the charger is turned off in this case. Therefore, the power loss $P_{c}$ is given by:

$$
P_{c}=P_{c}^{o n} \cdot x_{c}=\left(P_{c d c t}+P_{s w}+P_{c t r l}\right) \cdot x_{c},
$$

where $x_{c}$ is a binary variable with the interpretation that $x_{c}=1$ if the charger is turned on and $x_{c}=0$ otherwise.

In the buck mode, the power loss components is given by:

$$
\begin{aligned}
P_{c d c t}= & I_{\text {out }}^{2} \cdot\left(R_{L}+D \cdot R_{s w 1}+(1-D) \cdot R_{s w 2}+R_{s w 4}\right) \\
& +\frac{(\Delta I)^{2}}{12} \cdot\left(R_{L}+D \cdot R_{s w 1}+(1-D) \cdot R_{s w 2}+R_{s w 4}+R_{C}\right), \\
P_{s w}= & V_{\text {in }} \cdot f_{s} \cdot\left(Q_{s w 1}+Q_{s w 2}\right) \\
P_{c t r l}= & V_{\text {in }} \cdot I_{\text {controller }},
\end{aligned}
$$

where $D=V_{\text {out }} / V_{\text {in }}$ is the PWM duty ratio (less than 1$)$ and $\Delta I=$ $V_{\text {out }} \cdot(1-D) /\left(L_{f} \cdot f_{s}\right)$ is the maximum current ripple; $f_{s}$ is the switching frequency; $I_{\text {controller }}$ is the current flowing into the controller; $R_{L}$ and $R_{C}$ are the equivalent series resistances of the inductor $L$ and capacitor $C$, respectively; $R_{s w i}$ and $Q_{s w i}$ are the turn-on resistance and gate charge of $i^{\text {th }}$ MOSFET switch in Figure 4, respectively.

In the boost mode, the power loss components are given by:

$$
\begin{aligned}
& P_{c d c t}= \\
& \left(\frac{I_{\text {out }}}{1-D}\right)^{2} \cdot\left(R_{L}+D \cdot R_{s w 3}+(1-D) \cdot R_{s w 4}+R_{s w 1}+D \cdot(1-D) \cdot R_{C}\right) \\
& +\frac{(\Delta I)^{2}}{12} \cdot\left(R_{L}+D \cdot R_{s w 3}+(1-D) \cdot R_{s w 4}+R_{s w 1}+(1-D) \cdot R_{C}\right) \\
& P_{s w}=V_{\text {out }} \cdot f_{s} \cdot\left(Q_{s w 3}+Q_{s w 4}\right), \\
& P_{c t r l}=V_{\text {in }} \cdot I_{\text {controller }},
\end{aligned}
$$

where $D=1-V_{\text {in }} / V_{\text {out }}$ and $\Delta I=V_{\text {in }} \cdot D /\left(L_{f} \cdot f_{s}\right)$.

\subsubsection{System Power Analysis}

As Figure 2 shows, current flows out of the power source into the CTI through the source-to-CTI converter, then it flows into chargers which connect the CTI and selected destination EES banks. Therefore, the source power $P_{s r c}(t)$ equals to the sum of the power loss of the source-to-CTI voltage converter $P_{c, s}(t)$, the power drain to the ground $P_{\text {waste }}(t)$, and the power delivered from the CTI to the inputs of charger, denote by $P_{c t i}(t)$, as follows:

$$
\begin{aligned}
P_{s r c}(t) & =V_{s r c}(t) \cdot I_{s r c}(t)=P_{c, s}(t)+P_{\text {waste }}(t)+P_{c t i}(t) \\
& =P_{c, s}(t)+P_{\text {waste }}(t)+\sum_{k=1}^{N} P_{i n, k}(t),
\end{aligned}
$$

where $P_{i n, k}(t)$ is the input power of the $k^{t h}$ charger from the CTI. For the $k^{\text {th }}$ charger, the relation between the input power $P_{i n, k}(t)$ and output power, denoted by $P_{\text {out }, k}(t)$, is given by:

$$
\begin{aligned}
& P_{\text {in }, k}(t)=V_{c t i}(t) \cdot I_{c t i, k}(t)=P_{\text {out }, k}(t)+P_{c, k}(t), \\
& P_{\text {out }, k}(t)=V_{d s t, k}^{C C}(t) \cdot I_{d s t, k}(t),
\end{aligned}
$$

where $V_{c t i}(t), I_{c t i, k}(t), V_{d s t, k}^{C C}(t)$ and $I_{d s t, k}(t)$ are the input voltage $V_{\text {in }}$, input current $I_{\text {in }}$, output voltage $V_{\text {out }}$ and output current $I_{\text {out }}$ of the $k^{t h}$ charger, respectively. Note both of $P_{c, k}(t)$ and $P_{c, s}(t)$ can be computed by (8) but using different sets of parameters. 
Furthermore, the output power of the $k^{\text {th }}$ charger equals to the sum of three power components, i.e., the power that the $k^{\text {th }}$ EES bank receives, denoted by $P_{d s t, k}(t)$, the power dissipation on the internal resistance of the EES bank, denoted by $P_{\text {int }, k}(t)$, and the power loss due to the rate capacity effect for battery banks, denoted by $P_{\text {rate }, k}(t)$. Details of these power components are shown in (13):

$$
\begin{aligned}
P_{\text {out }, k}(t) & =P_{\text {int }, k}(t)+P_{\text {rate }, k}(t)+P_{d s t, k}(t), \\
P_{\text {int }, k}(t) & =\left(V_{d s t, k}^{C C}(t)-V_{d s t, k}^{O C}(t)\right) \cdot I_{d s t, k}(t), \\
P_{\text {rate }, k}(t) & =V_{d s t, k}^{O C}(t) \cdot I_{d s t, k}(t) \cdot\left(1-\eta_{\text {rate }}\left(I_{d s t, k}(t)\right)\right), \\
P_{d s t, k}(t) & =V_{d s t, k}^{O C}(t) \cdot I_{d s t, k}(t) \cdot \eta_{\text {rate }}\left(I_{d s t, k}(t)\right),
\end{aligned}
$$

where $\eta_{\text {rate }}\left(I_{d s t}, k(t)\right)$ is the charging efficiency with consideration of the rate capacity effect.

Let $E_{\text {HEES }}(t)$ denote the energy stored in the HEES system (among all EES banks) at time $t$. Then we have:

$$
\frac{d}{d t} E_{H E E S}(t)=\sum_{k=1}^{N} P_{d s t, k}(t)-\sum_{k=1}^{N} P_{s d, k}(t),
$$

where $P_{s d, k}(t)$ is the self discharge power of the $k^{\text {th }}$ EES bank, and $P_{d s t, k}(t)=0$ if the $k^{t h}$ bank is not selected to be charged at time $t$.

\subsection{Charge Allocation Optimization Problem Formulation}

Let us consider a charge allocation process starting at time $T_{0}$ and ending at time $T_{0}+T_{a}$, where $T_{a}$ is the total time duration of the charge allocation process. Assume that at time $T_{0}$, we have knowledge of the SoCs of all EES banks, based on which we can determine the EES bank open circuit voltages $V_{d s t, k}^{O C}\left(T_{0}\right), \forall k \in S$. Also assume that the power source profile, i.e., $P_{s r c}(t)$ and $V_{s r c}(t)$, is given over the time period $\left[T_{0}, T_{0}+T_{a}\right]$. For the charge allocation problem, there are three sets of variables that we could control for optimizing the global charge allocation efficiency. The first is the CTI voltage $V_{c t i}(t)$ which can be controlled by the source-toCTI converter. The second is a subset $S^{\prime}(t) \subseteq S$ of destination EES banks selected among all $N$ EES banks to receive the energy from the power source. The third is the set of EES bank charging currents $\left\{I_{d s t, k}(t)\right\}, k \in S$ which can be controlled by the chargers. Note that if $k \notin S^{\prime}(t)$, i.e., the $k^{t h}$ EES bank is not selected, we have $I_{d s t, k}(t)=0$. Therefore, the global charge allocation (GCA) optimization problem can be formally described as follows:

Given: Initial OCVs of all destination EES banks, $V_{d s t, k}^{O C}\left(T_{0}\right)$, $\forall k \in S$, power source profile $P_{s r c}(t), V_{s r c}(t), \forall t \in\left[T_{0}, T_{0}+T_{a}\right]$.

Find: $V_{c t i}(t), S^{\prime}(t)$, and $I_{d s t}, k(t), \forall k \in S$ and $\forall t \in\left[T_{0}, T_{0}+T_{a}\right]$.

Maximize: the global charge allocation efficiency, defined by:

$\eta_{G C A}=\int_{T_{0}}^{T_{0}+T_{a}} \sum_{k=1}^{N}\left(P_{d s t, k}(t)-P_{s d, k}(t)\right) d t / \int_{T_{0}}^{T_{0}+T_{a}} P_{s r c}(t) d t$

or equivalently, maximize the total energy stored among all destination EES banks at time $T_{0}+T_{a}$ (at the end of charge allocation), given by $\int_{T_{0}}^{T_{0}+T_{a}} \sum_{k=1}^{N}\left(P_{d s t, k}(t)-P_{s d, k}(t)\right) d t$.

\section{Subject to:}

1) The EES bank charging current must be no less than 0 and no greater than a maximum current value $I_{\max , k}$, i.e.,

$$
0 \leq I_{d s t, k}(t) \leq I_{\max , k}, \forall t \in\left[T_{0}, T_{0}+T_{a}\right], \forall k \in S .
$$

2) The system energy constraints, from (8), (11) (13),

$$
\begin{gathered}
\sum_{k=1}^{N}\left(P_{\text {int }, k}(t)+P_{\text {rate }, k}(t)+P_{d s t, k}(t)+P_{c, k}(t)\right) \\
\quad+P_{c, s}(t) \leq P_{s r c}(t) \\
P_{c, k}(t)=P_{c, k}^{o n}(t) \cdot x_{c, k}(t)
\end{gathered}
$$

where $x_{c, k}(t)=1$ if $k \in S^{\prime}(t), x_{c, k}(t)=0$ if $k \notin S^{\prime}(t)$, for $\forall t \in\left[T_{0}, T_{0}+T_{a}\right]$.

3) The energy stored in EES bank cannot exceed its maximum energy capacity $E_{\max , k}$ at any time $t$, i.e.,

$$
E_{k}\left(T_{0}\right)+\int_{T_{0}}^{T_{0}+t}\left(P_{d s t, k}(\tau)-P_{s d, k}(\tau)\right) d \tau \leq E_{\max , k}
$$

$\forall t \in\left[T_{0}, T_{0}+T_{a}\right], \forall k \in S$, where $E_{k}\left(T_{0}\right)$ stands for the initial energy stored in the $k^{\text {th }}$ bank at time $T_{0}$.

Due to the existence of binary variables $x_{c, k}(t)$, the GCA optimization problem is a mixed-integer nonlinear programming (MINLP) problem which is NP-complete. Therefore certain approximations shall be made for near-optimal solution of the GCA problem.

\section{OPTIMIZATION METHOD}

Before solving the global charge allocation (GCA) optimization problem described in Section 3.2, we first consider the instantaneous charge allocation (ICA) problem, which seeks to optimize the instantaneous charge allocation efficiency at a specific time instance. The ICA problem can be seen as a special case of GCA problem with $T_{a} \rightarrow 0$, and we will provide the algorithm for solving the ICA problem and getting near-optimal solution in Section 4.1. Subsequently, we break the whole charge allocation process into a series of time slots and solve an ICA problem at each decision epoch with an additional time dependent constraints for charging currents of different types of EES banks. Finally, we obtain the near-optimal solution of GCA problem by collecting the nearoptimal solutions of all ICA problems in chronological order.

\subsection{Instantaneous Charge Allocation}

The ICA optimization problem is described as follows:

Given: At time $t$, the OCVs of all destination EES banks $V_{d s t, k}^{O C}(t)$, $\forall k \in S$, power source characteristics, $P_{s r c}(t)$ and $V_{s r c}(t)$.

Find: $V_{c t i}(t), S^{\prime}(t)$, and $I_{d s t, k}(t)$, for $\forall k \in S$ at time $t$.

Maximize: instantaneous charge allocation efficiency, defined by,

$$
\eta_{I C A}=\sum_{k=1}^{N}\left(P_{d s t, k}(t)-P_{s d, k}(t)\right) / P_{s r c}(t),
$$

or equivalently, maximize total power received by all EES banks $\sum_{k=1}^{N} P_{d s t, k}(t)$.

Subject to: constraints (16) and (17).

Similar to the original GCA problem, the ICA optimization problem is again an MINLP problem, which is NP-complete. The ICA problem becomes a convex optimization problem if the following three assumptions hold.

- The CTI voltage $V_{c t i}(t)$ is given at time $t$, instead of a variable to control. 
- The set $S^{\prime}(t)$ of selected EES banks is given at time $t$, or equivalently, $x_{c, k}(t)$ is given for $\forall k \in S$. Note that we only perform charge allocation optimization on the EES banks belonging to $S^{\prime}(t)$.

- The $\operatorname{CCV} V_{d s t, k}^{C C}(t)$ is given at time $t$ for $\forall k \in S^{\prime}(t)$, rather than a function of $I_{d s t, k}(t)$ as in (4) and (5).

We refer the ICA problem with the above three assumptions as the simplified instantaneous charge allocation (SICA) problem. For the SICA problem, the optimization variables are only the charging currents $\left\{I_{d s t, k}(t)\right\}, k \in S^{\prime}(t)$. The SICA problem has a concave objective function (19) to be maximized, with linear inequality constraints (16) and convex inequality constraints (17). Thus the SICA problem is a convex optimization problem and can be solved in polynomial time, using standard convex optimization tools such as [12]. Based on this, we propose our algorithm to solve ICA problem in an iterative manner. In this algorithm, for each subroutine with a given $V_{c t i}(t)$, we find the near-optimal ICA efficiency with that given $V_{c t i}(t)$ value, denoted by $\eta_{I C A}\left(V_{c t i}(t)\right)$, in an iterative manner. In each iteration we heuristically update the selection set $S^{\prime}(t)$, the CCV value $V_{d s t, k}^{C C}(t)$, and subsequently solve the SICA problem until we converge to the near-optimal $\eta_{I C A}\left(V_{c t i}(t)\right)$ value. By searching in the feasible region of $V_{c t i}(t)$ and repeating this subroutine for different $V_{c t i}(t)$ values, we finally obtain the nearoptimal solution of the original ICA problem. The searching of near-optimal control variable $V_{c t i}(t)$ can be accelerated by assuming quasi-concave property of optimal ICA efficiency over $V_{c t i}(t)$ and and applying effective search algorithm such as the ternary search to exploit the quasi-concavity property. This assumption can be validated by simulation results. The pseudo-code of our algorithm is given in Algorithm 1.

In Algorithm 1, the convergence of $S^{\prime}(t)$ and $V_{c t i}(t)$ at time $t$ can be proved, and experimental results show quick convergence of the variables $V_{d s t, k}^{C C}(t), \forall k \in S^{\prime}(t)$. The reason for the quick convergence is that typically the difference between the OCV $V_{d s t, k}^{O C}(t)$ and the $\mathrm{CCV} V_{d s t, k}^{C C}(t)$ is not significant for a destination EES bank, since if the EES bank has relatively large internal resistance (e.g., battery bank), the optimal charging current will be relatively small.

We also implement some enhancements to the algorithm framework shown in Algorithm 1 to accelerate the convergence rate, such as using pre-computed $V_{d s t, k}^{C C}(t)$ values in future iterations. Details of such extensions are not discussed in this paper.

\subsection{Long-Term Global Charge Allocation}

In this section, we consider the GCA problem for long term charge allocation with given or predictable source profiles during time $\left[T_{0}, T_{0}+T_{a}\right]$. The GCA problem can be solved in the discrete time space. The duration of each time slot may be in the order of several seconds or minutes; while the duration of the whole charge allocation process, given by $T_{a}$, may last for several hours. One intuitive idea would be to solve an ICA problem using Algorithm 1 at every decision epoch (the beginning of each time slot), find the near-optimal $V_{c t i}(t), S^{\prime}(t)$ and $I_{d s t, k}(t), \forall k \in S$ values and then keep the values unchanged within that time slot, assuming that the power source profile and SoCs of destination EES banks will not change significantly during each time slot. However, this idea may cause greedy decisions of mainly charging the high efficiency banks (i.e., the banks with little rate capacity effect and small internal resistance, e.g., supercapacitor banks) first, which may prove not optimal in the later stage, in the following two aspects.

- Since the volumetric energy densities of the supercapacitor banks are much lower than the battery banks, it is highly possible that the former will be fully charged at the very early

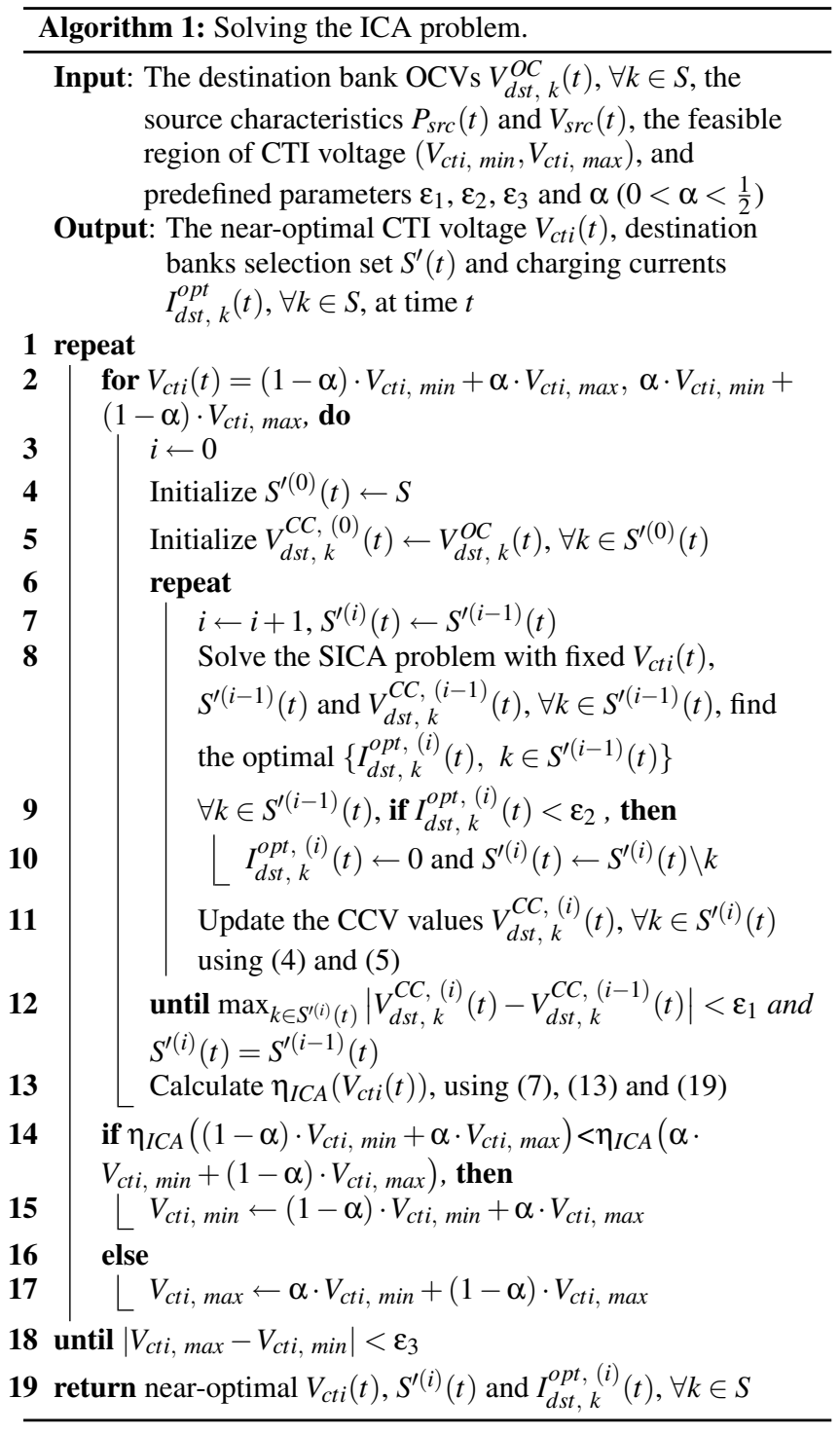

stage of the charge allocation process. If so, the system has to charge battery banks with large charging currents during the remaining charge allocation process, during which the ICA efficiency may drop significantly. Therefore the GCA efficiency over time period $\left[T_{0}, T_{0}+T_{a}\right]$ may not be optimal.

- The other reason comes from the self-discharge of supercapacitor banks. According to (7), the self-discharge rate $P_{s d, k}(t)$ is proportional to the square of $V_{d s t, k}^{O C}(t)$ for supercapacitor banks. Therefore if we charge the high efficiency banks with high rate in the early stage, the OCVs of such banks will grow rapidly, and we will suffer from more severe self-discharge in the later stage. In this way the GCA efficiency may also be affected.

The key idea to overcome the above two problems and let the charge allocation manager to "consider the future energy generation profile" is to impose a constraint on the total charging current of the supercapacitor banks. The constraint shall be time dependent, i.e., for higher power input $P_{s r c}(t)$, the constraint shall be looser. In this paper, we impose a time dependent upper bound on the total charging current of supercapacitor banks, denoted by $B_{s p c}(t)$. At time 
$t$, the bound is proportional to the input source power $P_{s r c}(t)$, i.e., $B_{s p c}(t)=\beta_{s p c} \cdot P_{s r c}(t)$. Therefore, the following constraint shall be added to the original ICA problem formulated in Section 4.1.

$$
\sum_{k \in S_{s p c}} I_{d s t, k}(t) \leq B_{s p c}(t),
$$

where $S_{s p c} \in S$ is the set of supercapacitor banks. We denote the ICA optimization problem with constraint (23) as the constrained ICA problem. Note that the constrained ICA problem can be solved in the similar way as the original ICA problem because the additional constraint is a linear inequality constraint. One effective way of setting the appropriate $\beta_{s p c}$ value for achieving near-optimal GCA efficiency is to charge the supercapacitor EES banks in such rate that they become nearly fully charged at the end of the whole charge allocation process.

Therefore, the near-optimal GCA optimization algorithm we proposed is summarized as follows. At time $T_{0}$, we globally considers the whole charge allocation process and determine the coefficient $\beta_{s p c}$ such that the supercapacitor banks will be (nearly) fully charged at time $T_{0}+T_{a}$. Then we break the whole charge allocation process $\left[T_{0}, T_{0}+T_{a}\right]$ into a series of time slots. At each decision epoch, we solve the constrained ICA problem, find the nearoptimal $V_{c t i}(t), S^{\prime}(t)$ and $I_{d s t, k}(t), \forall k \in S$ values of the constrained ICA problem and then perform charge allocation using the values unchanged within that time slot.

We made several improvements on the near-optimal GCA optimization algorithm to reduce the online computation costs, such as starting with the $S^{\prime}(t)$ and $V_{c t i}(t)$ from the previous decision epochs in the following ones, as long as the changes of the power source profile and SoCs of destination EES banks are within some certain thresholds, or using variable length time slots instead of fixed length ones according to the changes of source profile and destination bank SoCs.

\section{RESULTS AND DISCUSSION}

We consider two different HEES systems: one is consisting of four EES banks (two supercapacitor banks and two battery banks) and the other one is consisting of ten EES banks (four supercapacitor banks and six battery banks.) We first apply our proposed ICA optimization algorithm to these two HEES systems and show the improvements of the ICA efficiency, respectively. Then we consider two charge allocation processes (one lasts for 6 hours and the other lasts for 12 hours) and solve corresponding GCA problems using our proposed GCA optimization algorithm. The baseline setups we adopt in our experiment include: i) the equal power charging scheme (EPC, i.e., the input power is equally distributed into all EES banks), ii) battery banks first scheme (BBF, i.e., the input power is equally distributed into all battery banks), and iii) supercapacitor banks first scheme (SBF, i.e., the input power is equally distributed into all supercapacitor banks, and this scheme switches to BBF if all supercapacitor banks are fully charged). We use a constant CTI voltage for the baseline setups, during the whole charge allocation process. We report the ICA and GCA efficiencies obtained at several representative CTI voltage values. We use a Linear Technology LTM4607 converter as the charger and voltage converter models. The characteristics of the Li-ion battery is obtained by measuring and extracting the parameters of the battery model given in Figure 3. The characteristics of supercapacitor are obtained from Maxwell BCAP P270 series.

\subsection{Instantaneous Charge Allocation Problem}

Table 1 summaries the ICA efficiencies obtained by applying our proposed ICA optimization algorithm and baseline setups mentioned previously to the four banks HEES system, with the OCV of
Table 1: Comparison of ICA efficiencies for a HEES system with four banks.

\begin{tabular}{|c||c||c|c|c|c|}
\hline \multirow{2}{*}{$N$} & \multirow{2}{*}{ Near-optimal } & \multicolumn{4}{|c|}{ Baseline } \\
\cline { 3 - 6 } & & $V_{c t i}$ & $\mathrm{EPC}$ & $\mathrm{SBF}$ & $\mathrm{BBF}$ \\
\hline \multirow{3}{*}{4} & $15 \mathrm{~V}$ & $71.3 \%$ & $71.8 \%$ & $54.4 \%$ \\
\cline { 3 - 6 } & \multirow{3}{*}{$90.1 \%$} & $12 \mathrm{~V}$ & $73.1 \%$ & $72.4 \%$ & $54.7 \%$ \\
\cline { 3 - 6 } & & $10 \mathrm{~V}$ & $74.2 \%$ & $72.7 \%$ & $54.7 \%$ \\
\cline { 3 - 6 } & $8 \mathrm{~V}$ & $75.1 \%$ & $72.7 \%$ & $54.6 \%$ \\
\cline { 3 - 6 } & & $5 \mathrm{~V}$ & $75.0 \%$ & $70.7 \%$ & $54.0 \%$ \\
\hline
\end{tabular}

Table 2: Comparison of ICA efficiencies for a HEES system with ten banks.

\begin{tabular}{|c||c||c|c|c|c|}
\hline \multirow{2}{*}{$N$} & \multirow{2}{*}{ Near-optimal } & \multicolumn{4}{|c|}{ Baseline } \\
\cline { 3 - 6 } & & $V_{c t i}$ & EPC & SBF & BBF \\
\hline \multirow{3}{*}{10} & $15 \mathrm{~V}$ & $74.1 \%$ & $80.3 \%$ & $51.5 \%$ \\
\cline { 3 - 6 } & \multirow{3}{*}{$90.5 \%$} & $12 \mathrm{~V}$ & $74.4 \%$ & $80.4 \%$ & $51.6 \%$ \\
\cline { 3 - 6 } & & $10 \mathrm{~V}$ & $74.5 \%$ & $80.3 \%$ & $51.6 \%$ \\
\cline { 3 - 6 } & & $8 \mathrm{~V}$ & $74.5 \%$ & $80.0 \%$ & $51.6 \%$ \\
\cline { 3 - 6 } & & $5 \mathrm{~V}$ & $72.3 \%$ & $75.8 \%$ & $50.7 \%$ \\
\hline
\end{tabular}

$8 \mathrm{~V}, 2 \mathrm{~V}, 3 \mathrm{~V}$, and $6 \mathrm{~V}$, respectively. The instantaneous power of the PV array is $40 \mathrm{~W}$. The results show that the proposed algorithm achieves $15.0 \%$ to $36.1 \%$ efficiency improvement. Table 2 shows the results for the ten banks HEES system, with the OCV of $8 \mathrm{~V}, 8$ $\mathrm{V}, 1 \mathrm{~V}, 1 \mathrm{~V}, 4 \mathrm{~V}, 4 \mathrm{~V}, 3 \mathrm{~V}, 3 \mathrm{~V}, 3 \mathrm{~V}$, and $3 \mathrm{~V}$, respectively. The PV array provides instantaneous power of $60 \mathrm{~W}$. Our proposed algorithm can improve the ICA efficiency by $10.1 \%$ to $39.8 \%$. The significant improvement comes from the full utilization of the high efficiency bank(s). For example, the charging current for the supercapacitor bank with OCV of $8 \mathrm{~V}$ is much higher than those of the other three EES banks in the four banks HEES system.

Since the optimal CTI voltage $V_{c t i}$ depends on the power source characteristics, charge allocation scheme, and SoC and properties of EES banks, there is no way to determine a generally optimal $V_{c t i}$. From Tables 1 and 2, a fluctuation of the ICA efficiency up to $4.6 \%$ can be observed by setting different $V_{c t i}$ values. Hence, it is not surprising that an inappropriate $V_{c t i}$ can be often used in practice. In contrast, our proposed ICA optimization algorithm can search and converge rapidly to the near-optimal $V_{c t i}$.

\subsection{Global Charge Allocation Problem}

In the GCA problem, a PV array with a given power profile is used as the power source. We assume that the solar irradiance profile $G$ is given as a sine function of time $t$ from sunrise to sunset with the peak achieved at noon [13]. We employ the maximum power point tracking (MPPT) or maximum power transfer tracking (MPTT) [14] techniques for the PV array and achieve the optimal solar energy harvesting. In other words, we maximize the source power generation $P_{s r c}(t)$, by tracking the optimal source voltage $V_{s r c}(t)$ and current $I_{s r c}(t)$, based on the instantaneous variation of the solar irradiance, shading, temperature at time $t$. Therefore, both of $P_{s r c}(t)$ and $V_{s r c}(t)$ are given in our experiment. We consider the same HEES systems as in Section 5.1 but the initial OCVs of all the supercapacitor banks are set to $1 \mathrm{~V}$, assuming they are preemptive before a long-term charge allocation process.

Tables 3 and 4 list the GCA efficiencies of the target HEES systems with four EES banks and ten EES banks, respectively. Based on the experimental results, the GCA efficiency depends on the selection of charge allocation scheme, the power source profile, and the properties of EES banks. Although not performing well in the experiments, BBF is a reasonable charge allocation scheme for a long-time storing purpose, considering the small selfdischarge rate of the batteries. The results show that no baseline setup can consistently outperform the other baseline setups. In contrast, our proposed near-optimal GCA optimization algorithm can always achieve a near-optimal GCA efficiency by adaptively setting 
Table 3: Comparison of 6 hours process GCA efficiencies for a HEES system with four banks.

\begin{tabular}{|c||c||c|c|c|c|}
\hline \multirow{2}{*}{$N$} & \multirow{2}{*}{ Near-optimal } & \multicolumn{4}{|c|}{ Baseline } \\
\cline { 3 - 6 } & & $V_{c t i}$ & EPC & SBF & BBF \\
\hline \multirow{3}{*}{4} & \multirow{3}{*}{$83.8 \%$} & $15 \mathrm{~V}$ & $74.6 \%$ & $69.8 \%$ & $32.4 \%$ \\
\cline { 3 - 6 } & & $12 \mathrm{~V}$ & $74.9 \%$ & $69.7 \%$ & $32.4 \%$ \\
\cline { 3 - 6 } & & $10 \mathrm{~V}$ & $74.8 \%$ & $69.3 \%$ & $32.5 \%$ \\
\cline { 3 - 6 } & & $8 \mathrm{~V}$ & $74.4 \%$ & $70.4 \%$ & $32.6 \%$ \\
\cline { 3 - 6 } & & $5 \mathrm{~V}$ & $71.7 \%$ & $68.0 \%$ & $33.1 \%$ \\
\hline
\end{tabular}

Table 4: Comparison of 12 hours process GCA efficiencies for a HEES system with ten banks.

\begin{tabular}{|c||c||c|c|c|c|}
\hline \multirow{2}{*}{$N$} & \multirow{2}{*}{ Near-optimal } & \multicolumn{4}{|c|}{ Baseline } \\
\cline { 3 - 6 } & & $V_{c t i}$ & EPC & SBF & BBF \\
\hline \multirow{3}{*}{10} & \multirow{3}{*}{$82.6 \%$} & $15 \mathrm{~V}$ & $70.6 \%$ & $74.0 \%$ & $46.9 \%$ \\
\cline { 3 - 6 } & & $12 \mathrm{~V}$ & $71.0 \%$ & $73.8 \%$ & $47.0 \%$ \\
\cline { 3 - 6 } & & $10 \mathrm{~V}$ & $71.2 \%$ & $73.7 \%$ & $47.1 \%$ \\
\cline { 3 - 6 } & & $8 \mathrm{~V}$ & $70.9 \%$ & $72.9 \%$ & $47.2 \%$ \\
\cline { 3 - 6 } & & $5 \mathrm{~V}$ & $68.5 \%$ & $70.6 \%$ & $47.2 \%$ \\
\hline
\end{tabular}
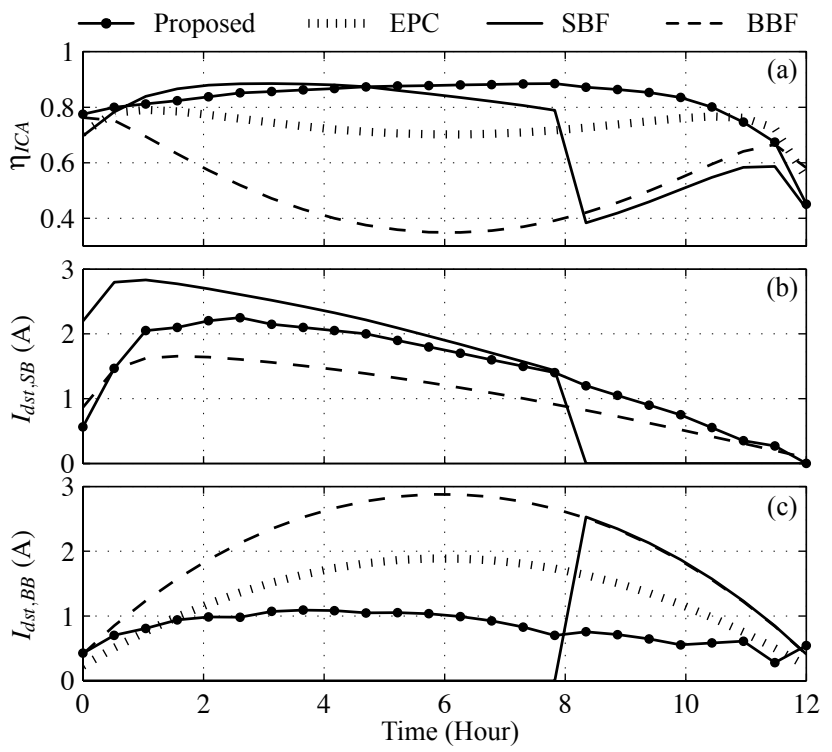

Figure 5: Comparison of ICA efficiencies, charging currents of one supercapacitor bank and one battery bank applying different charge allocation schemes during 12 hours for a ten bank HEES system.

$V_{c t i}(t)$ and selecting destination banks $S^{\prime}(t)$, and properly allocating charging currents $I_{d s t, k}(t)$ among all selected EES banks. For the four banks HEES system, the source power is a sine function with peak at $50 \mathrm{~W}$ and lasts for 6 hours. The improvement is from $8.9 \%$ to $51.4 \%$. For the ten banks HEES system, we extend our charge allocation process to a day (i.e., 12 hours using a PV array with peak power $100 \mathrm{~W}$ ) and the improvement is from $8.6 \%$ to $35.6 \%$.

Figure 5(a) shows the ICA efficiency by applying different charge allocation schemes versus time during the 12 hours charge allocation process for the ten banks HEES system. The solid curve with big dots describes the results of our proposed GCA algorithm, while the solid, dashed and dotted curves stand for that of the SBF, $\mathrm{BBF}$ and EPC, respectively. Figures 5(b) and 5(c) shows the charging current of one supercapacitor bank $\left(I_{d s t}, S B\right)$ and one battery bank $\left(I_{d s t}, B B\right)$ in the HEES system, respectively. The aggressive charge allocation scheme such as SBF can achieve a high ICA efficiency at early stage. However, after all the supercapacitor banks are fully charged (about 8 hours), SBF has to be switched to BBF, which has a much lower ICA efficiency (even lower than normal $\mathrm{BBF}$ due to the severe self-discharge of the fully charged supercapacitor banks.) Hence, SBF cannot guarantee the optimal GCA efficiency. For conservative charge allocation schemes such as EPC and BBF, are not able to achieve optimality either because of relatively large rate capacity effect and un-fully utilization of the high efficiency EES banks.

\section{CONCLUSIONS}

Hybrid electrical energy storage (HEES) system is one of the most promising and practical ways to achieve a high performance and low-cost EES system. This is the first paper that introduces fundamental concepts of global charge allocation (GCA), including the system architecture and formal problem definition. The GCA problem is formulated as a mixed-integer non-linear optimization problem. We propose a systematic algorithm for the GCA problem by solving a series of instantaneous charge allocation (ICA) at each decision epochs with time dependent constraints to avoid greedy decisions which proved not optimal. Furthermore, we propose an effective way of solving the ICA problem and getting near-optimal solution in an iterative manner, where in each iteration we solve a convex optimization problem which can be solved by standard convex optimization algorithm in polynomial time. We perform experiments using a photovoltaic array as the power source and demonstrate that the proposed algorithm outperforms the baseline setup by an improvement of $8.6 \%$ to $51.4 \%$ in GCA efficiency.

\section{REFERENCES}

[1] H. T. Odum, "Energy quality and carrying capacity of the earth," Tropical Ecology, 1975.

[2] J. Baker and A. Collinson, "Electrical energy storage at the turn of the millennium," Power Engineering Journal, 1999.

[3] T. Moore and J. Douglas, "Energy storage, big opportunities on a smaller scale," EPRI J., 2006.

[4] D. H. Doughty, P. C. Butler, A. A. Akhil, N. H. Clark, and J. D. Boyes, "Batteries for large-scale stationary electrical energy storage," ESI, 2010.

[5] M. Pedram, N. Chang, Y. Kim, and Y. Wang, "Hybrid electrical energy storage systems," in ISLPED, 2010.

[6] F. Koushanfar, "Hierarchical hybrid power supply networks," in $D A C, 2010$.

[7] H. Chen, T. N. Cong, W. Yang, C. Tan, Y. Li, and Y. Ding, "Progress in electrical energy storage system: A critical review," Progress in Natural Science, 2009.

[8] M. Chen and G. Rincon-Mora, "Accurate electrical battery model capable of predicting runtime and I-V performance," IEEE T. on Energy Conversion, 2006.

[9] D. Shin, Y. Wang, Y. Kim, J. Seo, M. Pedram, and N. Chang, "Battery-supercapacitor hybrid system for high-rate pulsed load applications," in DATE, 2011.

[10] D. Linden and T. B. Reddy, Handbook of Batteries. McGrew-Hill Professional, 2001.

[11] Y. Choi, N. Chang, and T. Kim, "DC-DC converter-aware power management for low-power embedded systems," IEEE T. on CAD, 2007.

[12] M. Grant and S. Boyd, "CVX: Matlab software for disciplined convex programming, version 1.21." http: / / cvxr.com/cvx, Feb. 2011.

[13] Measurement and Instrumentation Data Center. www. nrel.gov/midc.

[14] Y. Kim, N. Chang, Y. Wang, and M. Pedram, "Maximum power transfer tracking for a photovoltaic-supercapacitor energy system," in ISLPED, 2010. 